\title{
Study of the inter-particle necks in selective laser sintering
}

\author{
Famasp Fhabvala, Eric Boillat and Rémy Glardon \\ Laboratoire de Gestion et Procédés de Production, Swiss Federal Institute of Technology (EPFL), Lausanne, Switzerland
}

\begin{abstract}
Purpose - Since pulsed lasers are mainly used in selective laser sintering (SLS) - contrarily to selective laser melting (SLM) - only the exterior of the powder particles is molten while their core stays solid. The purpose of this paper is to investigate the binding mechanism between two particles of titanium powder.

Design/methodology/approach - A dedicated experimental setup is used to isolate the particles. They are then irradiated by the laser. SEM micrographs are taken at each step and image analysis is performed. The obtained results are compared with the predictions of a thermal model allowing for the incorporation of the latent heat of fusion and for a realistic surrounding. The absorbed laser intensity is modeled by means of the Mie theory.

Findings - The growing of the interparticular necks and the volume of liquid formed for different repetition rates are measured and compared with numerical simulations. A good agreement is found. A new method to easily find the absorption coefficient of the laser into the grain and the heat exchange coefficient with the exterior is developed.

Originality/value - This paper leads to a better understanding of the necking phenomena involved in the SLS consolidation process. An experimental set-up has been developed to observe and quantify the final state of a small amount of laser sintered grains. This process has been shown to be replicable and trustful. The thermal model leads to good predictions of the particle final sintering state.
\end{abstract}

Keywords Titanium, Sintering, Lasers, Selective laser sintering, Simulation, Inter-particle necks

Paper type Research paper

\section{Introduction}

The selective laser sintering (SLS) process is limited by two antagonist processes. If too much energy is given, all the material melts and part precision is lost. On the other hand, if the particles do not receive enough energy, the bonding between the particles is weak and the parts have poor mechanical properties. The understanding of the molten phase evolution and of its influence on the inter-particle necks evolution is essential to play between these two limitations.

In the SLS process, pulsed lasers are mostly used. Since only the surface of the powder particles is molten, consolidation can be achieved at much lower average power. Liquid bridges appear between the powder particles and are responsible for the bonding. The mechanical properties of the final part depend on the shape of these bonds. The goal of this study is to understand how the evolution of the liquid bridges (also called necks) is influenced by the laser parameters.

The idea is to collect enough experimental information concerning the sintered state of a few particles under different laser conditions. The comparison of these results with the liquid phase evolution (predicted by the thermal model)

The current issue and full text archive of this journal is available at www.emeraldinsight.com/1355-2546.htm

Rapid Prototyping Journal

19/2 (2013) 111-117

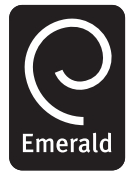

[DOI $10.1108 / 13552541311302969$ ] leads to a better understanding of the binding mechanisms involved in SLS.

This work is divided into two parts. The first one is an experimental study. The geometrical features of a few particles are analyzed after different types of laser treatment corresponding to different values of the power, repetition rate and pulse duration. These results are obtained thanks to the development of an adequate system for the separation and the transport of the two particles. A П-shaper is also mounted on the laser, so that the Gaussian shape of the beam becomes a top-hat profile. The evolution of the inter-particle neck is then obtained by image processing on electron micrographs.

The experimental results are used to clarify the exact relationships between the melting kinetics and the bounding mechanisms. They should be a keystone in developing a comprehensive model of the SLS process taking the thermal phenomena and the flow of molten material into account.

In the second part of this work a thermal model of the interaction between the laser beam and a single (metallic) grain is presented. This model demonstrates the deep influence of the laser parameters, like power, pulse duration and repetition rate, onto the melting kinetics and the liquid phase behavior. The experimental results are compared to the thermal model output.

The authors would like to thank Mélanie Dafflon and Benoit Lorent at LSRO2 (EPFL) for their important involvement in the Delta3 robot experiments. They are also thankful to the Interdisciplinary Centre for Electron Microscopy (EPFL) for providing access to electron microscopes.

Received: 7 July 2011

Revised: 23 December 2011

Accepted: 21 March 2012 


\section{State of the art}

\subsection{Consolidation process}

The global consolidation process is essentially driven by the minimization of the total surface energy (German, 1996). Under laser heating, the material wants to group in a sphere. When too much energy is given, giants balls tend to form inside the part (balling effect) (Klocke and Wagner, 2003; Tolochko et al., 2004).

The kinetics of the material reorganization is slowed down by viscous effects and material re-solidification. Because of these two phenomena, the control of the quantity of molten material and of its lifetime is essential to ensure a strong microscopic bounding and a good macroscopic precision of any part made by SLS (Fischer et al., 2003b). The quantity of liquid and the time it takes to get back to solid state can be influenced by the laser parameters. It can be shown that metallic particles essentially undergo superficial melting under short laser pulses (Fischer et al., 2003a).

\subsection{Thermal model}

There are several thermal models describing the laser-matter interactions (Fischer et al., 2003b; Kolossov, 2005). They are usually based on unrealistic assumptions about the latent heat of fusion which is often neglected and about the thermal exchange between the grain and the exterior (insulated (or single) grain model). These assumptions all result in considerable simplifications of the numerical model.

\section{Experimental setup}

The investigated material is pure titanium powder. The radii are distributed between 45 and $50 \mu \mathrm{m}$. Two methods are used.

\subsection{Method A: isolation of two particles}

Two particles of powder are deposited on copper substrates. Copper has been chosen because of its high thermal conductivity. In this way, the observation should not be modified by an unwanted melting of the substrates. A network of holes is created on the substrate to avoid the particles to be blown away over long distances when they are hit by the laser. Each hole has a depth and a diameter of $300 \mu \mathrm{m}$.

To isolate two titanium particles, a high precision robot, the Delta3 robot (Dafflon et al., 2006), is used. It has been developed in the LSRO2 lab at EPFL (Figure 1).

It is based on parallel kinematics with three degrees of freedom and built with flexure hinges and non contact actuators and sensors allowing movements without friction. It presents a bandwidth of $400 \mathrm{~Hz}$ and stroke of $4 \mathrm{~mm}$ in all directions with a position repeatability of $\pm 10 \mathrm{~nm}$. This compact robot fits in a cube of $210 \mathrm{~mm}$ side.

Manipulation of beads was operated with a glass pipette (Figure 2) with a tip diameter of 5-15 $\mu \mathrm{m}$. Picking operations are carried out using vacuum. But releasing such micro-parts is difficult because gravity becomes negligible compared to adhesive forces: by interrupting the vacuum, objects still remain on the glass tip. The vibration of the pipette is an efficient solution to release the objects once they are touching their final position. The vibration can be induced by a piezoelectric element (square signal of $2 \mathrm{kHz} @ 0.3-0.6 \mathrm{~V}$ ) at the settling point.

The powder particles are deposited into the holes of the substrate. Although the two particles are smaller than the holes diameter, they are held in contact by electrostatic forces. After that they are irradiated by the laser under inert gas (argon).
A given amount of energy is brought to the system by the laser and a SEM micrograph is taken afterwards. The cycle is then repeated:

1 irradiation of the particles; and

2 micrograph analysis.

In this way pictures of the bonding of two particles are obtained and the neck formation can be precisely observed.

3.2 Method B: observation of particles on a powder bed A П-shaper is mounted in the laser. This optical element transforms the laser Gaussian beam in a top-hat profile. In this way, the exact energy delivered to the particles is known.

Powder beds of titanium are made and irradiated with different scanning speeds of the laser. Knowing the spot radius of the laser $(\omega=0.1 \mathrm{~mm})$, the exact time of illumination is known. In the case of continuous mode, a preheating of the powder has to be taken into account (Section 1). For each speed, a SEM micrograph is taken. Then the cycle:

- irradiation of the particles; and

- micrograph analysis is repeated.

This manipulation is appropriate to quantitative measurements, while the previous one (Section 1) essentially leads to qualitative observations of the bonding phenomena.

\subsection{Measurements on micrographs}

Different fixed stages of the consolidation process are obtained. Electron micrographs are suitable because of their large depth of field.

To compute the energy given to the particles, the power of the laser and the irradiation time at each step is measured precisely.

The electron micrographs are analyzed with a Labview program: Imaq vision builder. It measures the quantities listed in Table I.

From this data, indirect values describing the sintering process can be computed by simple geometry (Table II).

The volume of liquid contributing to the neck is assumed to be the volume of two spherical caps of height $\mathrm{br}_{1} /\left(\mathrm{r}_{1}+\mathrm{r}_{2}\right)$ and $\mathrm{br}_{2} /\left(\mathrm{r}_{1}+\mathrm{r}_{2}\right)$ for the particles of radius $\mathrm{r}_{1}$ and $\mathrm{r}_{2}$, respectively, (Figure 3).

The lateral surface $s$ of the system is the surface of the two particles minus the indented surface. The sintering rate $S$ characterizes the surface minimization. It is the ratio $100\left(s_{0}-s\right) /\left(s_{0}-s_{\text {min }}\right)$ where $s_{\text {min }}$ is the surface of one single sphere and $s_{0}$ the surface of two separate spheres with the same volume of material (Figure 4). A similar parameter has been defined in Klocke and Wagner (2003). The difference is that they relate it to the radii of the particles while it is related to the surface of the system in this paper.

\section{Results}

\subsection{Experiments}

Three different pairs of particles are studied, each at a different repetition rates $(10,20$ and $50 \mathrm{~Hz})$. Each of them has been irradiated two times with increasing laser power. The observed behaviors are illustrated by the pictures of Figure 5. These pictures have been obtained by using Method A (Section 3.1).

This method essentially gives qualitative data but since the beam is multimodal, the laser energy absorbed by the particles 
Figure 1 The Delta3 robot

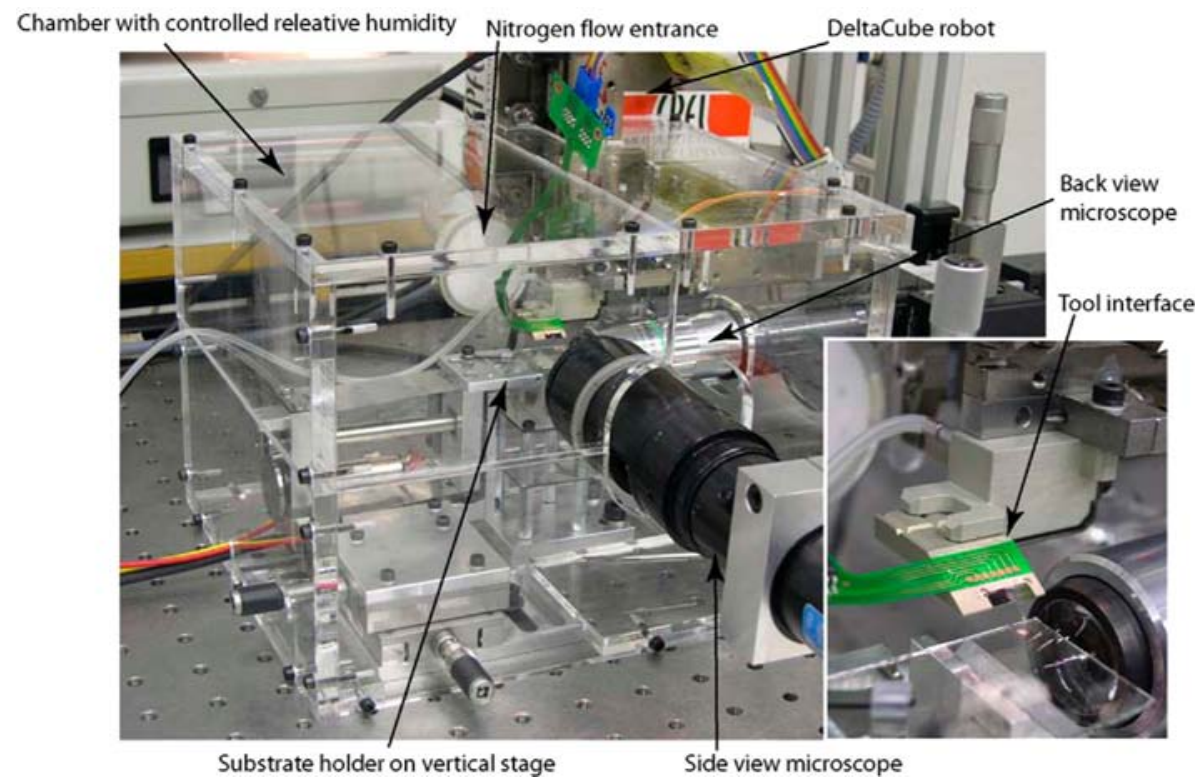

Source: From Dafflon et al. (2006)

Figure 2 The pipette

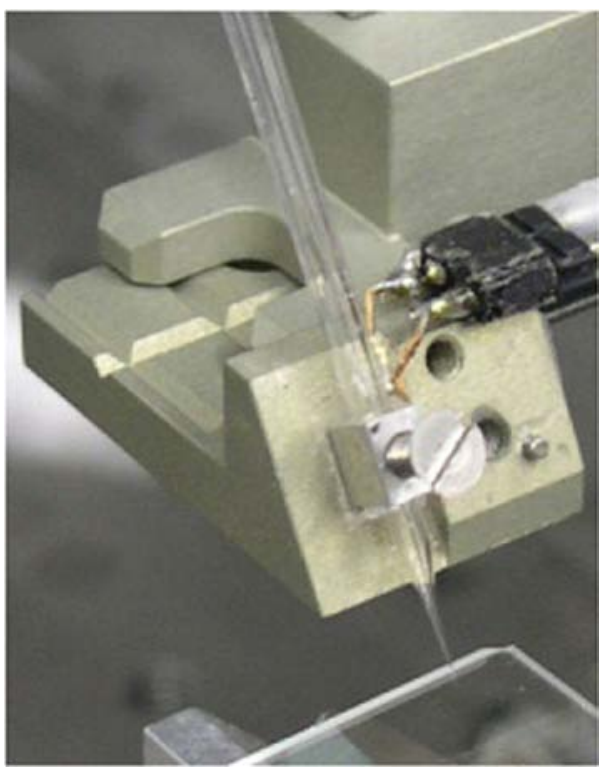

Table I Direct measurements from Imaq vision builder

\begin{tabular}{lc}
\hline Measured quantities & Symbol \\
\hline Particles radii & $r_{1}, r_{2}$ \\
Neck diameter & $a$ \\
Particles centers displacement & $b$
\end{tabular}

is not easy to compute. Quantitative data can only be derived by applying Method B (Section 3.2).

The measurements presented on Table III are made using Method B and directly obtained from the Imaq vision builder. In all cases, the irradiation time is $10 \mathrm{~ms}$, the laser power is
Table II Geometrical characteristics of a sintered system

\begin{tabular}{lc}
\hline Characteristic & Symbol \\
\hline Lateral surface of the system & $S$ \\
Neck volume & $V$ \\
Sintering rate & $S$
\end{tabular}

Figure $32 \mathrm{D}$ representation of the pair of particles

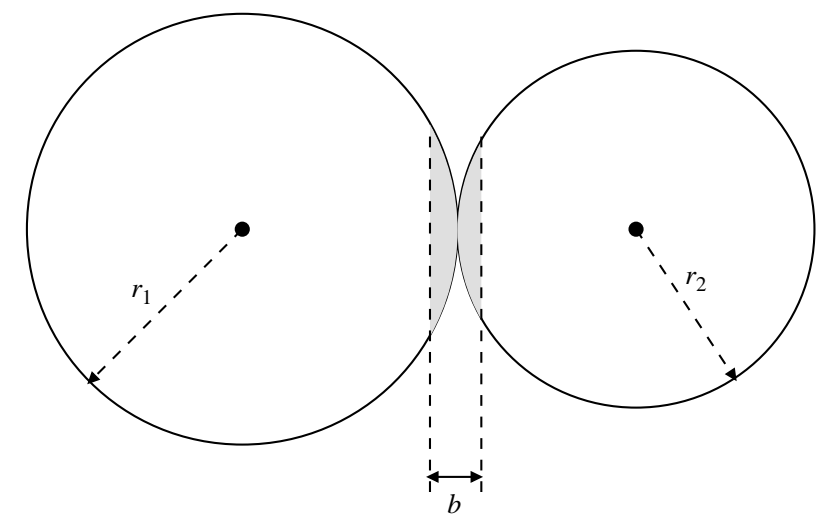

Figure 4 Sintering rate

$$
\mathrm{S}=0 \%
$$$$
0 \%<\mathrm{S}<100 \%
$$$$
\mathrm{S}=100 \%
$$
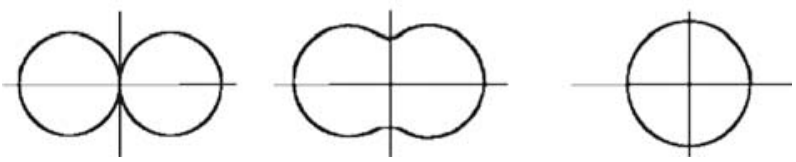

Source: Image modified from Klocke and Wagner (2003) 
Figure 5 Particles before (a) and after (b) (c) irradiation

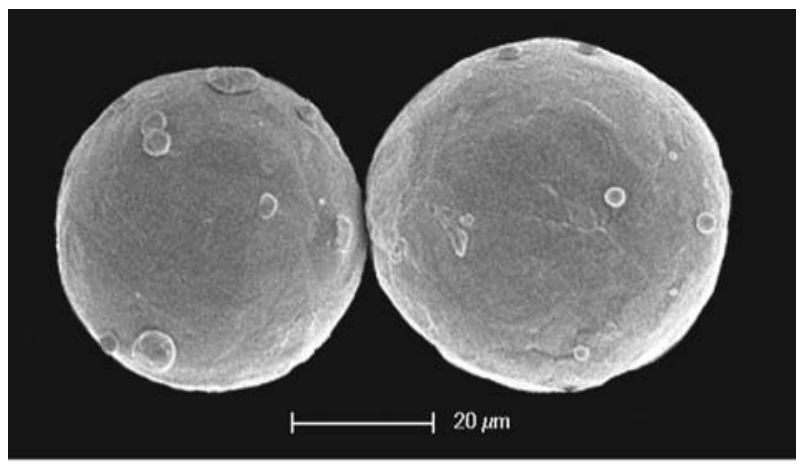

(a)

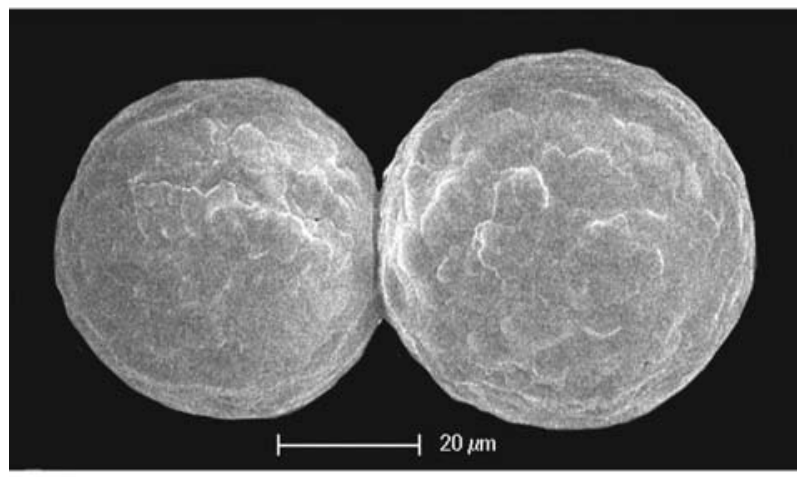

(b)

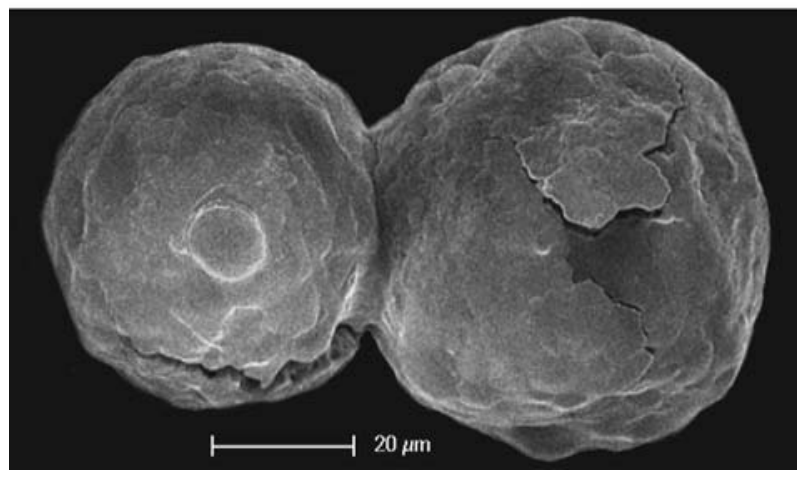

(c)

Table III Data directly deduced from Imaq vision builder

\begin{tabular}{rrrrrr}
\hline & $\mathrm{E}(\mathrm{mJ})$ & $r_{\mathbf{1}}(\boldsymbol{\mu m})$ & $\boldsymbol{r}_{\mathbf{2}}(\boldsymbol{\mu m})$ & $\boldsymbol{a}(\boldsymbol{\mu m})$ & $\boldsymbol{b}(\boldsymbol{\mu m})$ \\
\hline $10 \mathrm{kHz}$ & 0 & 45 & 49 & 0 & 0 \\
& 20 & 45 & 50 & 56 & 23 \\
$20 \mathrm{kHz}$ & 40 & 45 & 50 & 76 & 28 \\
& 0 & 40 & 48 & 0 & 0 \\
& 18 & 40 & 48 & 57 & 3 \\
$50 \mathrm{kHz}$ & 36 & 40 & 47 & 58 & 5 \\
& 0 & 41 & 42 & 0 & 0 \\
& 16 & 41 & 42 & 60 & 1 \\
& 32 & 41 & 41 & 62 & 4 \\
\hline
\end{tabular}

$4.5 \mathrm{~W}$ and the associated pulse duration is $200 \mathrm{~ns}$. The beam radius of the laser is $100 \mu \mathrm{m}$ and the total energy, $E$, irradiated on the particles is calculated for each step with an uniformly distributed intensity due to the П-shaper.
The measurements obtained directly from the Imaq vision builder are summarized in Table III.

The lateral surface of the system $s$, the neck volume $V$ and the sintering rate $S$ (Table II) are found by processing the data of Table III. The results of these computations are summarized in Table IV.

As expected, the lateral surface decreases and the volume of the neck increases from one step to the other. This phenomenon is even more marked for the $10 \mathrm{kHz}$ repetition rate, because the quantity of involved liquid is bigger.

More precise observations (Method A) of the sintering process between the two grains show that the first stage of the inter-particle necks was not homogeneous. Many little bridges are formed and hold the particles together (Figure 6(a)). Then the entire neck forms and grows as expected (Figure 6(b)).

After irradiation the formation of droplets on the surface of the particles is observed, as pointed out in Huppmann and Riegger (1975), which means that a part of liquid formed cannot contribute to the inter-particle neck.

\subsection{Numerical model and simulations}

A thermal model is developed to describe the interaction between a laser beam and a (spherical) grain.

This model consists of a pde for the temperature $T$ and the enthalpy $u$ per unit mass inside the grain. It reads:

$$
\rho \partial_{t} u-k \Delta T=0 \text {, in the grain }
$$

with $\rho$ the density, $k$ the thermal conductivity of the material and $\Delta$ the Laplace operator. The algebraic relation $T=\beta(u)$ between the temperature and the enthalpy allows for a nonvanishing latent heat of fusion. The equation (1) is completed by a boundary condition for the heat flux $\varphi$ (measured in W/ $\mathrm{mm}^{2}$ ) entering the grain:

$$
\varphi=\kappa\left(T_{0}-T\right)+I_{\mathrm{dir}}+\alpha \frac{P}{\pi \omega^{2}}, \text { on } g r \text {. bndry. }
$$

The first term on the right hand-side models the heat exchange between the grain and the exterior. In this case, the exterior is considered as a heat sink of fixed temperature $T_{0}$ and fixed linear heat exchange coefficient $\kappa$. The second term $I_{\text {dir }}$ is the electro-magnetic intensity coming directly from the laser. The last term, $I_{\text {refl, }}$ represents the electromagnetic intensity entering the grain after multiple reflections onto the neighboring grains. Since the multiple reflection process is linear and has no preferred direction, $I_{\text {refl }}$ is constant and in proportion with laser intensity. If $P$ denotes the laser power and $\omega$ the spot size, one has $I_{\text {refl }}=\alpha P / \pi \omega^{2}$ for a absorption coefficient $\alpha$.

Table IV Processed data

\begin{tabular}{lrrrc}
\hline & $E(\mathrm{~mJ})$ & $S\left(\mu \mathrm{m}^{2}\right)$ & $\mathrm{V}\left(\mu \mathrm{m}^{3}\right)$ & $S(\%)$ \\
\hline $10 \mathrm{kHz}$ & 0 & $55,321.0$ & 0.0 & 0.0 \\
& 20 & $49,998.0$ & $36,586.0$ & 47 \\
& 40 & $48,506.0$ & $53,188.0$ & 61 \\
$20 \mathrm{kHz}$ & 0 & $49,015.0$ & 0.0 & 0.0 \\
& 19 & $48,230.0$ & 630.0 & 8 \\
$50 \mathrm{kHz}$ & 38 & $46,499.0$ & $1,708.0$ & 26 \\
& 0 & $43,187.0$ & 0.0 & 0.0 \\
& 16 & $43,030.0$ & 65.0 & 2 \\
& 32 & $41,727.0$ & $1,014.0$ & 22 \\
\hline
\end{tabular}


Figure 6 Necking phenomena

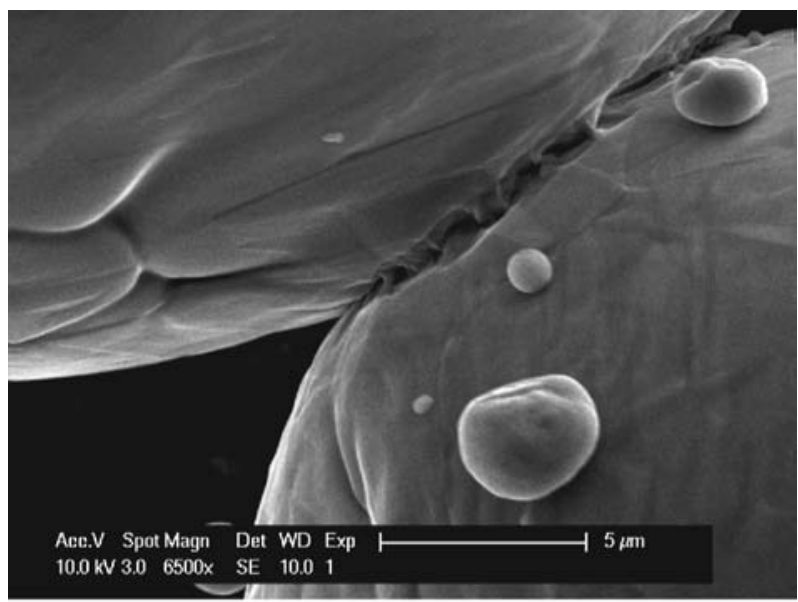

(a) Neck after firrst irradiation

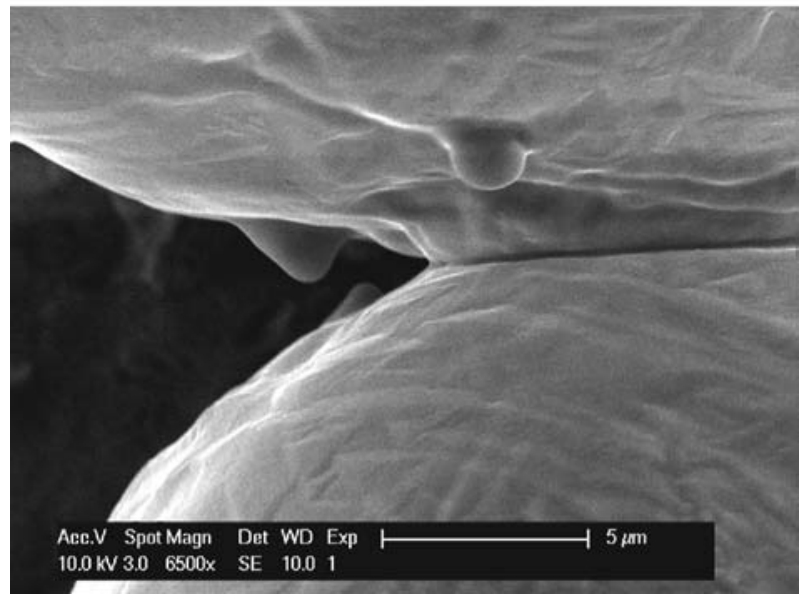

(b) Neck after second irradiation

The evolution equations (1) and (2) can be solved from a given initial condition for the enthalpy by the Chernoff scheme (Magenes et al., 1987) with adequate time steps and mesh sizes. The idea is to use this numerical technique to demonstrate how the laser parameters influence the molten material behavior. The examples presented below (Section 3) consider a titanium grain. The grain radius $r$ is $50 \mu \mathrm{m}$ and the physical properties of bulk titanium are summarized in Table $\mathrm{V}$.

The laser is a Nd:YAG operating at a power $P=4.5 \mathrm{~W}$ (power used in the following experiments). It is used either in continuous mode or in pulsed mode (pulse duration: $200 \mathrm{~ns}$ ). Because of the $\Pi$-shaper, the beam is considered to have a top-hat profile with spot radius $\omega=0.1 \mathrm{~mm}$ (Section 3.2).

The four important entries of this model are actually on the right-hand side of the heat flux balance equation (2). They are the direct intensity $I_{\text {dir }}$, the heat sink temperature

Table V Physical properties of bulk titanium

\begin{tabular}{ll}
\hline Density $\rho$ & $4.5 \times 10^{-3}\left(\mathrm{~g} / \mathrm{mm}^{3}\right)$ \\
Specific heat $c_{p}$ & $0.528\left(\mathrm{~J} / \mathrm{g}^{\circ} \mathrm{C}\right)$ \\
Thermal conductivity $k$ & $17.0 \times 10^{-3}\left(\mathrm{~W} / \mathrm{mm}^{\circ} \mathrm{C}\right)$ \\
Melting temperature $T_{m}$ & $1,660\left({ }^{\circ} \mathrm{C}\right)$ \\
Latent heat of fusion $L$ & $435.4(\mathrm{~J} / \mathrm{g})$ \\
\hline
\end{tabular}

$T_{0}$ and the coefficients $\alpha, \kappa$. For the first two, the following remarks can be made:

1 If experiments using Method B (Section 3.2) are considered, the laser is drawing lines and $T_{0}$ allows for a preheating of the powder. A temperature:

$$
T_{0}=500^{\circ}
$$

is used. This temperature is justified by macro simulations of the powder bed (Kolossov, 2005; Jhabvala et al., 2010; Jhabvala, 2010; Tolochko et al., 2003) showing that the observed particles are preheated before they are irradiated. If experiments using Method A (Section 3.1) are simulated, no preheating has to be considered (the powder has time to cool down between each pulse) and $T_{0}$ can be set to ambient temperature:

$$
T_{0}=T_{\mathrm{amb}} .
$$

2 At the grain scale, the top-hat laser beam can be considered as a plane wave and the theory developed by Mie (1908) is used to solve Maxwell equations. The output are the electrical field $\mathbf{E}$ and the magnetic displacement $\mathbf{H}$ inside the particle. The absorbed intensity $I_{\text {dir }}$ can be expressed as a triple vector product:

$$
I_{\mathrm{dir}}=-(\mathbf{E} \times \mathbf{H}) \cdot \mathbf{n}
$$

with $\mathbf{n}$ the unit normal to the grain boundary $\partial \%$. The total power $P_{\text {dir }}$ absorbed by the grain is the integral of $I_{\text {dir }}$ over the surface $\partial \%$. It can be shown that:

$$
\int_{\partial \%} I_{\mathrm{dir}} \mathrm{d} \sigma=\gamma P \frac{r^{2}}{\omega^{2}}
$$

with $P$ the laser power, $\omega$ the spot radius and $\gamma$ a constant only depending on the dielectric constant of the material and on the radius $r$ of the spherical particle. In case of a titanium grain of radius $r=50 \mu \mathrm{m}$ one has:

$$
\gamma \simeq 48 \% \text {. }
$$

After these remarks, it only remains to determine $\alpha$ and $\kappa$. An original method has been developed. It is presented in the following section.

\subsubsection{Determination of $\alpha$ and $\kappa$ (continuous mode)}

In the case of a continuous laser, the temperature solution to equations (1) and (2) can be assumed to be homogeneous in the $50 \mu \mathrm{m}$ radius titanium particle. The Biot number, $B i=\kappa \mathrm{r} / 3 k$, determines whether or not the temperatures inside a body under a stationary heat source varies significantly in space. For the considered particle, $B i \ll 1$ which means that the homogeneous heating hypothesis can be made. In this case, the uniform grain temperature converges exponentially towards an asymptotic value $T_{\infty}$ with a time constant $t_{c}$ :

$$
T(t)=T_{\infty}+\left(T_{0}-T_{\infty}\right) e^{-\left(t / t_{c}\right)}
$$

The equilibrium temperature depends on $\alpha$ and $\kappa$. It is obtained by averaging the balance equation (2) over the grain boundary with zero heat flux $\varphi$ (at equilibrium). Since the power directly absorbed is given by equation (6), one gets:

$$
T_{\infty}=T_{0}+\frac{(\gamma+4 \alpha) P}{4 \kappa \pi \omega^{2}}
$$


The time constant is related to $\kappa$ and to the physical properties of the grain:

$$
t_{c}=\frac{\rho C_{p} r}{3 \kappa} .
$$

The idea to determine the values of $\alpha$ and $\kappa$ is to make two experiments with different powers $P_{1}$ and $P_{2}$ and to measure the respective times $t_{1}^{*}$ and $t_{2}^{*}$ when the melting temperature $T_{m}$ is reached. Using equations (8)-(10) a system of two equations is found for the two unknowns $\gamma+4 \alpha$ and $\kappa$ :

$$
\left\{\begin{array}{l}
T_{m}-T_{0}=\frac{(\gamma+4 \alpha) P_{1}}{4 \kappa \pi \omega^{2}}\left(1-e^{-\left(3 \kappa t_{1}^{*} / \rho C_{p} r\right)}\right) \\
T_{m}-T_{0}=\frac{(\gamma+4 \alpha) P_{2}}{4 \kappa \pi \omega^{2}}\left(1-e^{-\left(3 \kappa t_{2}^{*} / \rho C_{p} r\right)}\right)
\end{array}\right.
$$

The numerical values used for $\mathrm{P}_{1}$ and $\mathrm{P}_{2}$ are $5.8 \mathrm{~W}$ and $4.2 \mathrm{~W}$, respectively. The laser spot is $\omega=0.1 \mathrm{~mm}$. The transition between the situation where no particles are fused and the situation where molten material appears turns to be quite sharp. In this case, $t_{1}^{*} \simeq 30 \mathrm{~ms}$ and $t_{2}^{*} \simeq 60 \mathrm{~ms}$, respectively. The heat sink temperature $T_{0}$ is chosen as in equation (3).

Finally the absorption coefficient $\alpha$ for the multiple reflection and the heat transfer coefficient $\kappa$ are found by solving equation (11) (Boillat, 2011) and by taking the value (equation (7)) of $\gamma$ into account:

$$
\alpha=3 \% \quad \kappa=0.013 \mathrm{~W} / \mathrm{mm}^{2} \mathrm{~K}
$$

\subsubsection{Stability of the identification}

The identification (equation (12)) for $\alpha$ and $\kappa$ is stable with respect to measurement errors. The consequence of relative errors $\mathrm{d} \tau / \tau$ and $\mathrm{d} n / n$ in the measurement of the ratios $\tau=t_{2}^{*} / t_{1}^{*}$ and $n=P_{1} / P_{2}$ is a relative variation $\mathrm{d} \kappa / \kappa$ for the heat transfer coefficient $\kappa$. The estimate:

$$
\mathrm{d} \kappa / \kappa \leq C\left(\frac{\mathrm{d} n}{n}+\frac{\mathrm{d} \tau}{\tau}\right)
$$

has been obtained in Boillat (2011) for a constant $C$ depending on the characteristic time $t_{c}$ (equation (10)). If $\tau=t_{2}^{*} / t_{1}^{*} \geq 2$, the formula is:

$$
C=\frac{t_{c}}{t_{1}^{*}} \frac{n^{2}}{n-1} .
$$

For the considered situation, (Table $\mathrm{V}$ and equation (12)), $t_{c} \simeq 3 \mathrm{~ms}, t_{1}^{*} \simeq 30 \mathrm{~ms}$ and $n=5.8 / 4.2 \simeq 1.38$ therefore $C \simeq 0.5$.

\subsubsection{Grain simulations in pulsed mode}

In the case of pulsed laser, homogeneous heating of the grain can no longer be assumed, since the heat source is not constant in time. Instead of using equations (8)-(11) and equations (1) and (2) have to be solved with the coefficients $\alpha$ and $\kappa$ taken in (12) and with an absorbed intensity $I_{\text {dir }}$ as in equation (5). The heat sink temperature $T_{0}$ is set to ambient value equation (4).

Since particles are assumed to be spherical, the generalized coordinates $(r, \theta, \phi)$ are well adapted. The absorbed intensity $I_{\text {dir }}$ has a special dependency with respect to the polar angle $\phi$ (Mie, 1908) and the 3D thermal problem can be reduced by a standard truncated Fourier analysis. The result is a finite number (one for each Fourier mode) of coupled and nonlinear 2D heat equations in the $(r, \theta)$ plane[1].

Figure $7(\mathrm{~b})$ and (c) represents the time evolution of the ratio between the volume of the molten material and the volume of the grain for different repetition rates.

For these two simulations the laser power is $4.5 \mathrm{~W}$. With the same power the whole particle stays nearly solid in continuous mode (Figure 3). But in pulsed mode liquid appears almost immediately. The grain is partially fused but has time to cool down completely between pulses. At $2 \mathrm{kHz}$ repetition rate, the volume of liquid reaches 18 percent of the total particle volume at each pulse. This behavior is not favorable to good sintering. With a lot of liquid formed at the same time balling can occur. Consequently, a repetition rate of $10 \mathrm{kHz}$ or more seems suitable to get good bonding between the particles. In the following experiments the selected repetition rates will be in the range $10-50 \mathrm{kHz}$.

\subsection{Comparison between simulation and experiments} For the $10 \mathrm{kHz}$ frequency, the simulations (Figure $7(\mathrm{~b})$ ) predict that 6 percent of the grain is molten after $10 \mathrm{~ms}$ irradiation. In the related experiment, the neck volume is found to be 4 percent of the entire material for the first laser illumination, and 6 percent for the second. The simulations are in agreement with the experiments and good predictions about the final stage of the bonding of the particles for different laser parameters are provided by the thermal model.

To predict exactly the amount of liquid contributing to the neck a flow model describing the motions of the molten material would be needed (Anestiev and Froyen, 1999). Capillary driven flow models unfortunately lead to complex free surface problems (Shikhmurzaev, 1997) and simplifying hypotheses need to be made.

\section{Conclusion}

This paper leads to a better understanding of the necking phenomena involved in the SLS consolidation process. An experimental set-up to observe and quantify the final state of a couple of laser sintered grains has been developed.

Figure 7 Evolution of the quantity of liquid formed for different repetition rates

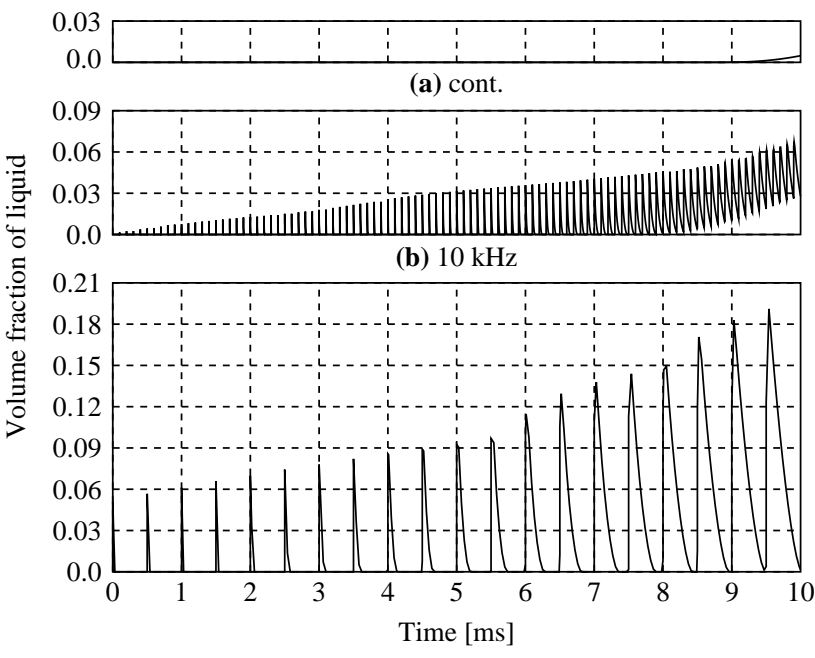

(c) $2 \mathrm{kHz}$ 
This process has been shown to be replicable and trustful. To interpret the experimental data, the time evolution of liquid is simulated by using a thermal model of the laser-matter interaction. A new method to find easily the absorption coefficient $\alpha$ of the laser into the grain and the heat exchange coefficient $\kappa$ with the exterior is developed. The thermal model leads to good predictions of the particle final sintering state.

The main issue is then to explain the capillary flow mechanisms leading from the molten material to the neck formation. This analysis should ideally result in a comprehensive SLS model combining the thermal description and an adequate (and possibly not too complex) fluid flow model.

\section{Notes}

1 For the Nd:YAG fundamental radiation.

2 The coupling of the $2 \mathrm{D}$ heat equations in the $(r, \theta)$ plane is due to the non-vanishing latent heat.

\section{References}

Anestiev, L.A. and Froyen, L. (1999), "Model of the primary rearrangement processes at liquid phase sintering and selective laser sintering due to biparticle interaction", Fournal of Applied Physics, Vol. 86 No. 7, pp. 4008-17.

Boillat, E. (2011), "Détermination des coefficients de transfert et d'absorption", LGPP Internal Archives.

Dafflon, M., Lorent, B. and Clavel, R. (2006), "A micromanipulation setup for comparative tests of microgrippers", paper presented at ISR06, Münich.

Fischer, P., Romano, V., Weber, H.P., Karapatis, N.P., Boillat, E. and Glardon, R. (2003a), "Sintering of commercially pure titanium powder with a Nd:YAG laser source", Acta Materialia, Vol. 51 No. 6, pp. 1651-62.

Fischer, P., Leber, H., Romano, V., Weber, H.P., Karapatis, N., André, C. and Glardon, R. (2003b), "Microstructure of near infrared pulsed laser sintered titanium samples", Applied Physics A, Vol. 78 No. 8, pp. 1219-27.

German, R.M. (1996), Sintering Theory and Practice, Wiley, New York, NY.

Huppmann, W.J. and Riegger, H. (1975), "Modelling of rearrangement processes in liquid phase sintering", Acta Mettalurgica, Vol. 23, pp. 965-71.
Jhabvala, J. (2010), "Study of the consolidation process under macro- and microscopic thermal effects in selective laser sintering and selective laser melting", PhD thesis, EPFL, p. 4609.

Jhabvala, J., Antignac, T., Boillat, E. and Glardon, R. (2010), "On the effect of scanning strategies in the selective laser melting process", Virtual and Physical Prototyping, Vol. 5 No. 2, pp. 99-109.

Klocke, F. and Wagner, C. (2003), "Coalescence behaviour of two metallic particles as base mechanism of selective laser sintering", CIRP Annals - Manufacturing Technology, Vol. 52 No. 1, pp. 177-80.

Kolossov, S. (2005), "Non-linear model and finite element simulation of the selective laser sintering process", $\mathrm{PhD}$ thesis, EPFL, p. 3178.

Magenes, E., Nochetto, R.H. and Verdi, C. (1987), "Energy error estimates for a linear scheme to approximate nonlinear parabolic problems", Mathematical Modelling and Numerical Analysis, Vol. 21 No. 4, pp. 655-78.

Mie, G. (1908), "Beiträge zur Optik trüber Medien, speziell kolloidaler Metallösungen", Ann. Phys., Vol. 25 No. 4, pp. 377-445.

Shikhmurzaev, Y.D. (1997), "Moving contact lines in liquidliquid-solid systems", fournal of Fluid Mechanics, Vol. 334, pp. 211-49.

Tolochko, N.K., Arshinov, M.K., Gusarov, A.V., Titov, V.I., Laoui, T. and Froyen, L. (2003), "Mechanisms of selective laser sintering and heat transfer in Ti powder", Rapid Prototyping fournal, Vol. 9 No. 5, pp. 314-26.

Tolochko, N.K., Mozzharov, S.E., Yadroitsev, I.A., Laoui, T., Froyen, L., Titov, V.I. and Ignatiev, M.B. (2004), "Balling processes during selective laser treatment of powders", Rapid Prototyping fournal, Vol. 10 No. 2, pp. $78-87$.

\section{Corresponding author}

Jamasp Jhabvala can be contacted at: jamasp.jhabvala@ epfl.ch 